\title{
Multilinguales
}

14 | 2020

Enseignement universitaire et professionnalisation : enjeux, questionnements et défis socio-économiques

\section{Mobilité et éthique de la dignité dans Trois femmes puissantes de Marie Ndiaye}

Mobility and ethics of dignity in Trois femmes puissantes of Marie Ndiaye

\section{Youcef Immoune}

\section{(2) OpenEdition}

\section{Journals}

Édition électronique

URL : https://journals.openedition.org/multilinguales/5915

DOI : 10.4000/multilinguales. 5915

ISSN : 2335-1853

Éditeur

Université Abderrahmane Mira - Bejaia

Référence électronique

Youcef Immoune, « Mobilité et éthique de la dignité dans Trois femmes puissantes de Marie Ndiaye », Multilinguales [En ligne], 14 | 2020, mis en ligne le 10 décembre 2020, consulté le 02 juillet 2021. URL : http://journals.openedition.org/multilinguales/5915; DOI : https://doi.org/10.4000/multilinguales. 5915

Ce document a été généré automatiquement le 2 juillet 2021. 


\title{
Mobilité et éthique de la dignité dans Trois femmes puissantes de Marie Ndiaye
}

\author{
Mobility and ethics of dignity in Trois femmes puissantes of Marie Ndiaye
}

Youcef Immoune

1 Le roman Trois femmes puissantes de Marie Ndiaye (Gallimard, 2009) est l'histoire, en trois récits distincts, de trois destins de femmes, dont les parcours dynamiques et tragiques les situent entre l'Occident (la France) et l'Afrique (le Sénégal). Norah, Fanta et Khadi Demba luttent, chacune selon ses moyens et en fonction de circonstances particulières, contre la violence sociale, l'incommunicabilité et l'exil forcé. Trainant derrière elles un lourd héritage de trauma d'enfance, de parents durs, voire criminels, d'inhibitions, elles luttent pour retrouver la paix et rétablir les relations avec autrui, en étant tiraillées entre le besoin d'affranchissement et l'asservissement qui les conditionnent par les vieux démons de leur existence.

Cette quête, fondée sur la mobilité et l'éthique, interroge leur Être et les situe dans la douleur par rapport à l'Autre et dans le monde. Elles empruntent le chemin de la France vers l'Afrique ou inversement de l'Afrique en direction de la France. Elle les fait rencontrer dans un entre-deux où s'expérimente la volonté d'advenir à l'existence dans la dignité humaine (interrogation éthique), en tant que femmes (discours autour du féminisme), dans la dynamique nécessaire d'un mouvement libérateur prometteur de changements et de transformations (la dimension de la mobilité).

2 Les trois femmes disent "non", dans un long et tragique processus de résilience où tentent de s'accomplir le pouvoir de la pensée (l'interrogation, la projection) et l'universalité de l'existence: mettre fin à l'oppression des femmes, faire advenir la femme dans sa nature et sa culture, en signe d'ultimes degrés d'estime de soi. Pour cela, la nécessaire mobilité, tant dans la pensée que dans l'espace géographique, participe au débat féministe en interrogeant les rapports intersexuels, le pouvoir du langage (de l'écriture), le pouvoir social et politique. Interrogations, telles qu'elles peuvent être 
envisagées dans la nécessaire "mise à distance " représentée par la migration, la métamorphose des corps, la distance et la proximité, le social et l'individu.

C'est cette articulation mobilité-féminisme-dignité que cette présente contribution tente de reconstituer à travers le premier récit (Norah) afin de saisir les ressorts selon lesquels la spatialité de la mobilité permet d'engendrer les conditions de l'affranchissement et de l'accès à soi. La reconstituer dans la cohérence d'une lecture éthique de la problématique du féminisme, telle qu'elle s'envisage dans la distance entre le réel et la conception, la dimension sociopolitique et la dimension universelle, entre l'unité et l'hétérogène.

En effet, Trois femmes puissantes peut, par bien des aspects, posés par Jacques Chevrier (2004), relever de ce qu'il appelle la « Migritude », rappelant la notion de mobilité et les ressorts psychologiques et comportementaux liés à ce phénomène. A. Coulibaly (2015) précise, en renvoyant, sous ce terme, aux «[...] littératures gravitant autour de la mobilité nouvelle.» (34). Cela, en précisant également qu'il s'agit, en littérature, de «[...] passage d'une écriture de l'héroïsme, qui est mythifiante, vers des textes de la démythification, textes de la survie en Occident.» (34). De là découlent des notions connexes signifiant la rupture nécessaire qui infléchit la production littéraire, pour suggérer, donc sur le mode de la secondarité (au-delà du premier degré, sens différé, sous-entendu), « [...] l'impertinence, l'outrecuidance, le crime de lèse-majesté et de frustrations ainsi que le persiflage à l'encontre des autorités des pays d'origine.» (43), en œuvre pour marquer la distance et l'entre-deux. J. Foucart (2010) rappelle la complexité de cette spatialité particulière en lui attribuant trois spécificités qui décrivent un processus à trois termes: le vide ou cette "[...] incapacité des individus à faire sens ensemble, ou bien la menace sourde d'un foyer d'événements toujours susceptibles de gerber en une catastrophe ou bien encore l'impuissance de faire du social avec du psychique et, réciproquement, du digital avec de l'analogique et inversement. » (8); le compartimentage comme " [...] enfermement dans un pôle, ce qui se manifeste par le rejet ou l'identification, la destruction ou la fusion.» (8); enfin, le passage qui signifie « [...] le mouvement, l'aller-retour, la bifurcation, le bricolage. » (8).

Entre l'Occident (la France) et l'Afrique, cette spatialité complexe ne peut être que hautement symbolique, impliquant le rapport au corps, particulièrement celui de la femme africaine, qui autorise des glissements. Francine Barthe-Deloizy (2010) met en exergue, dans le cadre de cette problématique de la mobilité et de la rencontre, les passages nécessaires entre une spatialité corporelle objectivée et rationalisée (Occident) et une spatialité africaine, ritualisée (transe et possession) où «La nature devient surnature.» (88) Cela renvoie à la possibilité expérientielle et conceptuelle d'une «[...] " sortie de corps" dans d'autres mondes.» (88) et, donc, «[...] l'Afrique, terre des ancêtres, le lieu où se trouvent encore les divinités. » (88).

5 À partir de là, la réflexion s'installe dans la problématique de l'Éthique, plus particulièrement comme le suggère Thierry Pech (2001) de l'Éthique de la relation et son articulation à celle de la dignité. L'homme digne est « [...] celui qui tient son rang d'homme parmi les hommes, c'est-à-dire en relation avec eux et sous leur regard.» E. Levinas, dans Totalité et infini (Livre de Poche, 1991-1971) institue l'altérité en l'écartant du prisme de la raison: "La raison parlant à la première personne ne s'adresse pas à l'Autre, tient un monologue.» (69). Sous le prisme plutôt de la relation, la dignité, comme le souligne Thierry Pech, ne relève pas du paradigme de l'essentialisme, mais de celui d'une «[...] recherche des signes qui décrivent cet ordre invisible. ", donc d'un processus interactionnel, "[...] une injonction de communication et de reconnaissance. ». Levinas parle, dans ce sens, 
de la fonction expressive du langage qui revient au fait qu'il « [...] maintient précisément l'autre à qui il s'adresse, qu'il interpelle ou invoque. » (70), en supposant "des interlocuteurs, une pluralité. » et dont le « commerce » nous dit-il « est l'éthique ».

6 Dans le même ordre d'idées, comme le précise Th. Pech, il s'agit d'une "dignité moderne» qui rompt avec tout héritage moral (Afrique) et tout «culte de l'autonomie » (Occident). La reconnaissance s'impose comme

«[...] concept unificateur des différents visages de la dignité. » qui suppose que l'humanité «[...] est avant tout la qualité d'un être de relation et que c'est dans cette relation (à autrui, mais aussi à soi-même) que doit être recherché, non la définition substantielle, mais le sens de la dignité, c'est-à-dire la direction ou l'horizon de justice auquel elle nous appelle. ». C'est cet "absolument étranger » dont parle Levinas qui « [...] seul peut nous instruire» (71).

C'est dans le cadre de cette mise en perspective conceptuelle que s'inscrit l'analyse qui suit, en laissant parler le texte, Trois femmes puissantes.

\section{Migration du retour : immigration rejetée et blessures ouvertes des abandonnés}

7 Le père de Norah, immigré en France, décide de quitter «leur mère et la France» (Ndiaye : 46) et ses deux filles, Norah et sa sœur, et de rentrer au Sénégal, "parti brusquement en emmenant Sony », son fils de 5 ans (Ndiaye : 46), contre l'avis de sa femme, «puisqu'il savait qu'il n'aurait jamais obtenu son accord " (Ndiaye : 46). Il laisse la femme et les filles «dans le désespoir dont [elles] ne s'étai[ent] jamais remise [s]». Le père, poussé par l'urgence de son insatisfaction de sa condition en France, « où il piétinait dans un médiocre emploi de bureau » (Ndiaye : 46), lie son sauvetage au destin d'un fils, et non des filles.

Il identifie son insatisfaction à la femme, épouse et filles, et en tire un ressentiment, celui d'avoir échoué. L'échec consiste ici en une illusoire prospérité : l'attente qu'il formule par ce mariage mixte est de pouvoir engendrer des filles "presque blanches", "des filles de bonne facture ». "Or, cela a échoué » (Ndiaye : 26), puisque les filles « avaient toujours eu, pour leur père, le défaut d'être trop typées », lui révélant "l'inanité de son mariage avec une Française » (Ndiaye : 25). On retrouve ici le fantasme de l'Africain qui ne peut justifier sa migration (de l'Afrique en France) que par l'espoir d'une prospérité professionnelle, d'une intégration sociale (être dans le groupe blanc, estime de soi et des autres envers lui) et de cette dynamique il engendre, ultime fruit, une descendance aux couleurs et aux contours de cette mixité heureuse. Cette "irrémédiable répugnance " est encore vivace même à l'encontre des filles de sa fille Norah, d'autant plus, selon lui "elles n'étaient très jolies ni l'une ni l'autre ", chez qui il perçoit "une tristesse austère, convenable, réprimée, dans laquelle elles vivaient » (Ndiaye : 50). L'échec rend injustifiable cette immigration. Dans ce contexte, où règne plutôt la volonté du père (Africain), ce sont les femmes (mère et filles, voire petites-filles) qui sont présentées comme les fruits gâtés d'une entreprise avortée. Le père leur fait porter le lourd fardeau de ses frustrations et elles sont abandonnées en le portant avec le ressentiment d'avoir eu affaire à un père " [qui] était ainsi, implacable et terrible» (Ndiaye : 50). Un père qui, par sa migration inverse (de France en Afrique), est à la recherche d'un idéal d'où elles sont exclues: "Sony était donc le seul fils de cet homme qui n'aimait ni n'estimait guère les filles » (Ndiaye : 25). 
9 Il les exclut d'un idéal moral et matériel qu'il souhaite atteindre en Afrique sans elles. Son retour en Afrique a été envisagé et réussi dans un accomplissement qui le conforte dans sa dignité d'aristocrate. Un homme «qui avait pris si grand soin de la pureté de son apparence " (Ndiaye : 20); un homme "altier et inquiet " qui "s'était parfumé qu'aux essences les plus chics", ne voulant jamais " exhaler sa véritable odeur!». Il règne, en maitre, sur son fief où tout le monde lui doit obéissance « comme lui avaient toujours obéi jeunes ou vieux autour de lui » (Ndiaye : 19). Un homme qui veille jalousement sur ses privilèges «avec un air d'appréhension si enfantin» (Ndiaye 23). Ă ce titre, Maurice Godelier (1978), dans une perspective anthropologique, signale, en référence aux formes primitives de la domination (avant la formation des sociétés et États organisés en classes), de formes de domination reposant sur le consentement, justifié dans le "paradigme de la légitimité » : «Il y a donc dans tout pouvoir un enjeu fondamental et une force fondamentale qui retiennent les choses « en ordre " qui est le consentement que les dominés apportent souvent à leur domination. Et ce consentement, ils l'apportent parce que l'état des choses leur apparaît légitime.»(23-24). Sa femme, française, et ses filles, nées en France, ne peuvent participer, selon lui, à ce projet. La seule réussite avec laquelle il devait rentrer en Afrique c'est bien son fils Sony. C'est en lui, moyennant une bonne éducation, qu'il place l'espoir de réussir sa descendance. Les Françaises, mères et filles, sont abandonnées aux valeurs de l'Occident en lequel il ne croit pas et qu'il méprise. Éducation à l'occidentale qui rend le discours de Norah inopérant auprès de lui puisqu'il met ses réflexions "au compte d'une sensiblerie typique et de son sexe et du monde dans lequel elle vivait et dont la culture n'était pas la sienne " (Ndiaye: 21). Norah, face à son père "aux passions froides, qui n'avait passé en France que quelques années » ne peut qu'être envahie, elle, au cœur "ardent et vulnérable», par ce "sentiment d'impuissance, de colère et de découragement [...] qui lui faisaient cogner du front contre les irrémédiables différences d'éducation. » (Ndiaye : 21).

L'Occident est marqué dans le discours du père par son caractère étranger et étrange. Cette étrangeté est cristallisée dans un préjugé tenace: celui de l'orgueil d'un mâle misogyne, conforté dans la supériorité de son sexe (genre), consacrant par là l'infériorité de la femme identifiable à "l'émotivité qu'il méprisait sans retenue", typiquement rattachée à un "Occident avachi et féminisé » (21-22). Son projet est ainsi et a contrario fondé sur une Afrique dynamique et virilisée, consacrant la scission entre les deux mondes : « Nous n'avons pas le même pays, les sociétés sont différentes, dirait-il à peu près, docte, condescendant (...) » (Ndiaye : 21). Sa femme et ses files auraient été un fardeau inutile, et les abandonner, selon lui, en France, est quelque part juste dans la mesure où l'Afrique ne pouvait correspondre à leur nature. Norah aura à confirmer cette vision auprès des deux autres filles qu'il a eues tard avec une jeune Africaine. Ces filles qui l'accueillent "par un regard implacable " permettant à Norah de se dire que "rien ni personne ne pouvait réformer un tel homme car il aurait fallu rien moins que lui arracher le coeur.» (Ndiaye : 29).

Comme dans un miroir, le père et la fille se renvoient le reflet d'un monde manichéen où se joue une tension de répulsion entre l'Afrique et la France ; répulsion qui éloigne le père en procédant à une migration de retour. 


\section{De l'Europe à l'Afrique : figure tutélaire et culte de l'ordre}

11 Norah reçoit une lettre dans laquelle son père l'en enjoint à le rejoindre pour une affaire grave : Sony a tué sa belle-mère, la jeune épouse de son père et mère de deux jeunes filles. Elle effectue le voyage et, lors de son séjour chez son père, elle se retrouve confrontée à la figure tutélaire et un processus de prise de conscience s'opère : prise de conscience du fantasme (culte) de l'ordre cultivé tout au long de sa socialisation en France qui la met aux prises à une altérité heurtée envers l'homme : son père et son compagnon. Norah, formée dans le culte de l'ordre qui définit son féminisme, se heurte néanmoins de façon paradoxale, à la peur et au sentiment d'impuissance qui hantent sa conscience tourmentée.

Elle part du sentiment d'accomplissement dans l'ordre et la volonté : « Elle s'était élevée à la seule force de sa résolution et elle avait trouvé sa place dans un cabinet d'avocats, elle avait mis Lucie au monde et acheté un appartement [...] 》 (Ndiaye : 77). Cette Vision des choses, elle l'instaure et la pousse à son paroxysme sur la base de principes éthiques qui prônent l'ordre, la détermination, le travail. Norah s'établit dans la vie en tant qu'ordonnatrice de son monde parisien. Elle acquiert son autonomie économique, en tant qu'avocate (après de longues et dures années d'instruction). Elle divorce d'un premier mari qui entravait sa liberté et son libre arbitre. Elle achète un appartement pour élever selon ses préceptes sa fille, Lucie. Elle introduit chez elle un compagnon et sa fille "qu'elle devait entretenir». Sur ce petit monde, elle exerce son autorité et son ordre, même aux moyens de "ruades" (Ndiaye: 35 ). Dans cette bulle, qu'elle croyait façonner selon sa volonté, en tant qu' "emblème intime et modeste de sa persévérance, de sa discrète réussite » (Ndiaye : 30), elle s'aperçoit, avec dépit, que ces êtres, qui gravitent autour d'elle et qui sont doués tout autant "qu'elle de volonté, déséquilibrent son bel édifice. Non sans reproches autodirigés (s'avouer, honte, méfiance), elle évolue avec le désagréable sentiment que ce petit monde est un fardeau, "accroché à son dos ", un élément destructeur qui participe de "l'anéantissement de son honorable existence ", un élément parasitaire, qu'elle condamne en vertu d'une valeur morale, l'honneur.

13 Cette menace est vécue dans l'exacerbation. Norah est présentée dans son unité illusoire perturbée par son environnement immédiat qui, lui, ne peut être qu'hétérogène contrecarrant sa vision unitaire du monde. Elle

"dut se défendre contre ce sentiment de catastrophe et de terrible désordre", « compenser les effets de cette grave erreur» (Ndiaye: 38), lorsqu'elle sentait «qu'elle était humiliée de sentir que s'était nouée dans son dos, dans l'aubaine de son absence, une telle entente entre Jakob et les enfants » (Ndiaye : 72), lorsqu'elle «se demanda soudain pourquoi elle avait toujours eu l'impression indéfinissable, quand ils faisaient l'amour, qu'il se forçait un peu, qu'il payait son dû, leur couvert et leur logis, à lui et sa fille » (Ndiaye : 70).

Elle ne peut que se résoudre à une " présence constante, vigilante, inquiète » (Ndiaye : 38). En cela, Norah finit par se rendre compte qu'elle ressemble à son père dans cette volonté d'être dans un égoïsme effrayant. Elle est l'«angoissante incarnation» de son père. De la même manière que son père les a quittés pour s'accomplir en Afrique et prospérer en tant qu'entrepreneur, Norah souhaite également « en finir précisément avec la confusion", "se débarrasser ", "chasser", " en finir " avec les siens, qui, en les accueillant, elle a « fait le désordre et l'égarement » (Ndiaye : 30 ). 
Cette confusion fait naître en elle un sentiment d'impuissance et le désordre habite son esprit. Dans sa volonté d'échapper, par la réussite, au dénigrement et au mépris de son père, elle n'arrive cependant pas à libérer une parole contestataire envers l'autorité morale de son père. Cette contestation n'est pas opérante dans son face-à-face avec son père. Ce n'est qu' « au loin» qu'elle peut lui faire des reproches dans l'intimité de ses pensées. Face à lui, elle est impuissante, résolue "de plier le genou ", "de n'oser rien lui dire » (20). La réduction au silence (absence ou impossible langage) est caractéristique de ce Pierre Bourdieu (1982) le "code secret» qui opère dans le processus de domination en se manifestant particulièrement dans l'acte d'intimidation, "violence symbolique qui s'ignore en tant que tel (36): "Tout permet de supposer que les instructions les plus déterminantes pour la construction de l'habitus se transmettent sans passer par le langage [...] » (37). De la même manière qu'elle voudrait, sans succès cependant, contester l'autorité du père, Norah, tout autant autoritaire, redoute, sous l'effet d'un miroir inversé, la résistance que lui opposerait sa petite famille qui lui renverrait son propre autoritarisme. Elle est confrontée à un problème éthique : comment aller contre sa petite famille, sans aller contre l'autoritarisme dont elle fait preuve, mais qu'elle conteste chez son père ? Elle ne peut résoudre le problème et se sent là aussi impuissante, "se trouvant lâche et indigne ", " dégoûtée d'elle-même »: quant aux « remarques acrimonieuses qu'elle ne pouvait ou n'osait adresser à Jakob, l'homme avec lequel elle vivait depuis un an »; quant au fait d'être "excédée qu'il [Jakob] cherchât toujours à lui attribuer le rôle de l'ennuyeuse ou de la méchante [auprès des enfants] ». (Ndiaye : 30 )

La confusion est aliénante à double titre : aliénation au père et aliénation à elle-même, une sorte de double du père. Loin de sa base confortable de sa vie parisienne, ce voyage en Afrique et la confrontation au père qu'il lui permet, lui révèle sa condition paradoxale, cette impasse qui renforce son sentiment d'impuissance: se trouver «lâche» et «indigne». Son monde construit dans la certitude de la rectitude qu'elle imposait en France, loin de la confrontation, s'écroule. Lors d'un diner autour de la table de son père, en compagnie de Jakob et les deux filles, son père lui rappelle sa lâcheté quand, quelques années auparavant elle avait effectué un voyage solitaire au Sénégal pour s'enquérir de la situation de son frère, elle a renoncé, l'abandonnant alors qu' « il n'était pas au mieux de sa forme » (Ndiaye : 87). Autant elle a nié le fait, autant elle se rend à l'idée que le père a raison d'elle, que c'était «tous ces principes qui étaient contestés, dans leur rigueur, leur éclat, leur âpreté» (86), qu'elle apparaît "d'autant plus coupable d'avoir exigé et prôné, dans la vie qu'ils menaient ensemble, une telle rectitude » (Ndiaye : 86).

Le père, en l'invitant à le rejoindre en Afrique et par là en autorisant cette confrontation, lui tend indirectement et malicieusement le piège dans lequel elle s'enlise et se révèle dans ce qu'elle peut abhorrer chez lui. Le même égoïsme, la même intransigeance qui pouvaient les conduire, dans des circonstances différentes certes, à être injustes envers les leurs et les abandonner.

\section{De l'Afrique talisman et retour en paix en Europe}

16 Norah retrouve en Afrique, un père sur le déclin, « [son] père, cet homme fini, [qui] brillait de mille feux livides» (Ndiaye: 41), impliquant son cher fils Sony dans l'assassinat présumé de sa jeune épouse. Il appelle sa fille Norah, l'avocate, pour le défendre. Cette 
histoire révèle la lâcheté du père et plonge Norah dans une réflexion mystique et éthique qui lui permettrait de se départir des démons qui l'habitent.

Le père, en emmenant son fils avec lui, comme le rappelle la narratrice «s'était engagé dans une lettre laissée sur la table de la cuisine à veiller sur l'enfant mieux encore que sur sa propre vie, que sur ses affaires et son ambition" (Ndiaye : 46). Une promesse qu'il n'a pas tenue et Norah ne manque pas de lui rappeler : " - Qu'est-ce que tu as fait de Sony? Tu devais prendre soin de lui ! [...]»; «- Quel acte ? Qu'est-ce qu'il a fait ? Oh, mon Dieu, tu devais t'occuper de lui, l'élever convenablement!» (Ndiaye : 45), sur un ton de reproche qui a une double valeur: celle d'une parole non tenue, à l'adresse du père ; celle d'un acte manqué par défaut en ce qui la concerne. Tout en reprochant à son père de ne pas avoir pris soin de son frère, elle regrette cet engagement raté dans la mesure où elle se dit qu'elle aurait pu prendre soin de lui, persuadée, à l'instar de sa mère, «que Sony aurait un brillant avenir, des chances qu'elle n'aurait peut-être pas réussi, elle, simple coiffeuse, à lui donner. » (Ndiaye : 46) Sony tombe amoureux de la jeune épouse de son père et de cette relation « incestueuse » naissent deux filles. Le père tue sa femme et la police arrête le fils. Ce dernier ne se résout pas à dénoncer son père qui à son tour, sans se résoudre à se dénoncer, appelle sa fille, Norah, pour le défendre et le sortir de prison. Norah est tenaillée par "le sentiment de scandale et d'incompréhension indignée [qui] lui revenait brûlant, suffoquant » (Ndiaye : 71) lorsque, à la lecture d'un article de presse rapportant le fait divers, elle découvre, en parfaite étrangère, la société africaine qui n'hésite pas à condamner la femme et la présenter comme " mauvaise ", tentatrice car "c'est elle qui l'a appâté » (Ndiaye : 71), à l'origine de l'écart du fils qui de toute façon "n'aurait pas osé poser les yeux sur la femme de son papa » (71), et du crime. Une société qui conforte le père dans sa résolution à faire condamner le fils pour pouvoir le défendre ensuite, plutôt que de prendre le risque du déshonneur. Elle entre dans un dialogue de sourds, avec son père, sidérée qu'il lui demande de sauver son fils (« Sony a besoin d'un avocat, c'est tout ce qui compte » (76), lui dira-t-il), en condamnant doublement sa femme : l'assassiner et souiller sa mémoire. Norah, l'avocate "habituée à défendre les cas des femmes mortes de cette façon » (Ndiaye : 68), est appelée à renier ses convictions, en cédant au chantage affectif, au nom de l'intérêt et de l'amour qu'elle porte à son frère lui aussi victime.

Cette situation est si complexe pour Norah que le discours prend une dimension fantasmagorique. Norah convoque les démons pour tenter de justifier la malédiction qui s'abat sur elle et son frère. Elle refuse de " prendre en pitié leurs bourreaux, fussent-ils souriants et doux, fussent-ils de malheureux garçons sur le ventre desquels un démon s'était assis quand ils avaient cinq ans. " (Ndiaye : 68). Acculée par son père à défendre son frère, elle se dit qu'il est " possible aussi que, le démon sur son propre ventre, elle n'eût pas encore réalisé qu'il était là, assis, guettant son heure. » (Ndiaye : 67). En effectuant ce voyage en Afrique, Norah découvre son propre échec. Longtemps se disant étrangère à l'Afrique, elle s'est forgée dans le culte de la rationalité (occidentale). En France, elle a voulu se constituer dans l'absence du père, dans l'absence du mari. En cela, elle s'est installée dans l'ordre individuel de la rationalité où, seule, par son caractère déterminé, elle régente son petit monde. La vie des siens est rythmée par les interdits qu'elle leur impose. Elle construit sa bonne conscience où l'ordonnancement logique des choses, des paroles, des gestes conduit au bien et au vrai.

20 En venant en Afrique et en s'ouvrant malgré elle au père, elle redimensionne sa réflexion dans la sphère symbolique du ventre. Cette matrice ou cet empire de l'âme, 
chez les Africains (Patrice Yengo, 2014), la place, à son corps défendant, dans la sphère de l'émotion, de l'amour et de la liberté. En Afrique, elle retrouve son frère, Sony, cet objet d'amour, son manque. Elle retrouve surtout son père. Ă défaut de le comprendre d'un point de vue strictement rationnel, elle ne peut que se résoudre à le comprendre que de façon viscérale. Être-ventre d'un pays-ventre, elle doit se débattre avec les maux indéfinis, les secrets et la parole ambivalente et ambigüe. Ce n'est qu'à ce prix qu'elle cherche pourquoi Sony ne dénonce pas son père et se constitue prisonnier à sa place. Ce n'est que dans ce contexte qu'elle compose avec un père, mi-homme mi-oiseau, perché en haut d'un flamboyant, qu'elle doit considérer comme le symbole du clan par qui se transmet l'amour fraternel et filial.

C'est à ces moments de désordre qu'il devient urgent de passer par les affects que se transmettent les ventres une fois que les démons, ces êtres maléfiques qui entravent la vie, se lèvent. Ainsi Norah est amenée à prendre ses responsabilités :

«Elle voulait fuir au plus vite cette maison funèbre, elle avait l'impression cependant qu'en ayant accepté d'y revenir et sut repérer l'arbre où perchait son père, elle avait engagé sa responsabilité trop avant pour détourner le regard et rentrer chez elle. » (Ndiaye : 41)

21 Norah n'envisage son retour "chez elle", cette fois-ci, que dans la mesure où elle s'avoue que l'Afrique est en elle. Elle est happée dans ce ventre où sont noués les liens claniques auxquels rappelle le père: " $-T u$ as dit une fois, je me souviens, que tu ne laisserais jamais tomber Sony, déclara son père.» (Ndiaye : 77). En l'enchaînant par la parole donnée, le chef de clan, sur le déclin et sa disparition inévitable, se retourne vers Norah et en cela

«elle l'avait déchargé de ce terrible poids qu'était l'emprisonnement de Sony et qu'il ne restait plus qu'à attendre qu'elle eût réglé la situation, comme si, la charge morale, elle l'avait endossée et qu'il en était délivré à jamais. » (Ndiaye : 85)

Le père, bien qu'ayant toujours méprisé la fille, il se résigne à l'idée que c'est elle qui est consacrée dans le système de société qui pourtant la rejette. Inversement, il perd son fils qui devient le protégé de la sœur comme il aurait pu être le sien. De son côté Norah, d'abord étrangère au système clanique de son père, de tribu, voire de l'Afrique, elle se résigne à représenter ce système, à en être le garant. Elle le devient quand elle décide d'assumer la responsabilité, "déterminée, elle, à le [Sony] sauver et ainsi à l'affronter loyalement » (Ndiaye : 92). La loyauté réside dans la dignité acquise qui l'installe comme le pendant de l'autorité du père, avec lequel elle peut «oser » lui dire les choses, dans l'unité retrouvée du ventre familial, à savoir la « maison ».

23 Pour conclure, on dira que ce récit nous renseigne de manière particulière sur le sort d'une femme qui construit son féminisme dans le sillage d'un père dont la double migration et la double territorialité l'installe dans une existence traumatique qui dans l'espace d'un va-et-vient entre l'Afrique et l'Occident l'amène à la déconstruction et à la reconstruction de sa pensée éthique, au centre de laquelle se pose la question de la dignité.

Dignité d'une femme, née dans le contexte d'une migration paternelle ratée et abandonnée par lui, qui se forge dans la détermination de se construire laborieusement dans le culte de l'ordre : rationalité, rigueur morale, travail, autonomie. Dans ce cadre, elle n'a pas conscience de son autoritarisme qu'elle exerce sur un monde qu'elle juge avoir construit et devant exister selon sa volonté et son libre arbitre. Elle se construit contre les préjugés culturels du père, méprisant à l'endroit de la femme et d'elle, incarnation de son échec, typée, laide, occidentalisée, selon lui. Une dignité adossée à 
une vengeance sociale, guidée par une éthique de l'ordre, intransigeante sur la voie de la réussite sociale. Dignité d'une femme, confrontée plus tard à la territorialité de l'Afrique, étrangère et étrange, et donc au père étranger et étrange lui aussi. Elle prend conscience de la menace du désordre qui ébranle ses certitudes et les fondements de sa vie. Après s'être confondue dans ses contradictions, sa vision du monde préalable, rationaliste et féministe dans la rigueur occidentale, est modulée en fonction d'une vision africaine, magique et viscérale, créant ainsi un entre-deux où sa vie future, à son retour en France sera plus sereine et en phase à une éthique morale plus supportable pour les siens.

La mobilité de Norah est ce va-et-vient entre l'Occident et l'Afrique qui la fera évoluer d'un manichéisme révolté et tourmenté à un syncrétisme plus paisible : son féminisme est dans ce sens réorienté vers l'amour et la transigeance nécessaire pour gagner le respect de tout le monde. Sa dignité est ainsi doublement acquise : la dignité d'une femme libre (à l'occidentale) et la dignité d'un dignitaire (à l'Africaine). Le ciment de cette réconciliation est la loyauté envers soi et les autres, qui brise la fatalité de la lâcheté.

\section{BIBLIOGRAPHIE}

Barthe-Deloizy, Francine, Les spatialités du corps : Des pratiques ordinaires aux expériences extrêmes., Géographie, Université Michel de Montaigne - Bordeaux III, 2010. fftel-00878586

Bonjour, Pierre, «La dignité humaine, philosophie, droit, politique, économie, médecine : À partir de l'ouvrage coordonné par Thomas De Koninck et Gilbert Larochelle. », Reliance, $\mathrm{n}^{\circ} 20$ (2), 2006, 85-92. doi :10.3917/reli.020.92.

Bourdieu, Pierre, Ce que parler veut dire, Fayard, 1982.

Chevrier, Jacques, « Afrique(s) -sur-Seine : autour de la notion de migritude », Notre librairie, $\mathrm{n}^{\circ} 155-156,2004,96-100$.

Coulibaly, A., « Littérature migrante subsaharienne : l'ethnoscopie littéraire comme expression de la mobilité des écrivains de la migritude. ", Études littéraires, n 46 (1), 2015, 31-49. Disponible sur [https://doi.org/10.7202/1035082ar] (consulté le 03/01/2020)

Foucart, J., « Entre-deux et passages. Essai de conceptualisation à partir de la complexité et de la transaction. ", Pensée plurielle, $\mathrm{n}^{\circ} 24$ (2), 2010, 7-12. Disponible sur [doi :10.3917/pp. 024.0007.] (consulté le 12/02/2020)

Godelier, Maurice, « Pouvoir et langage ». In : Communications, 28, Idéologies, discours, pouvoirs. 1978, 21-27. Disponible sur [doi : https://doi.org/10.3406/comm.1978.1417 https:// www.persee.fr/doc/comm_0588-8018_1978_num1_1417] (consulté le 27/02/2020)

Levinas, Emmanuel, Totalité et infini, Le livre de Poche, 1991 (Martinus NijhofT, 1971)

Ndiaye Marie, Trois femmes puissantes, Gallimard, 2009.

Pech, Thierry, « La dignité humaine. Du droit à l'éthique de la relation », Éthique publique [En ligne], vol. 3, n², 2001, mis en ligne le 15 mai 2016. Disponible sur [URL : http:// 
journals.openedition.org/ethiquepublique/2526; DOI : https://doi.org/10.4000/ethiquepublique. 2526 ] (consulté le 02/03/2020)

Yengo, Patrice, « Le ventre dans l'écriture de Sony Labou Tansi et Tchicaya U Tam'si », Continents manuscrits [En ligne], 1, 2014, mis en ligne le 22 avril 2014. Disponible sur [URL : http://

journals.openedition.org/coma/238; DOI : 10.4000/coma.238] (consulté le 20 septembre 2018)

\section{RÉSUMÉS}

Cette présente contribution participe d'une réflexion sur les rapports à la spatialité dans le discours, inscrits dans l'épistémologie de la pragmatique, sensible aux spatialités vécues. Articulant mobilité-éthique de la relation-dignité, l'analyse de Trois femmes puissantes de Marie Ndiaye révèle comment la spatialité de l'Afrique et de l'Europe, dans le déplacement migratoire, est construction, négociation de sens et libération de la souffrance originelle.

This present contribution is part of a reflection on the relationships to spatiality in the discourse, inscribed in the epistemology of pragmatics, sensitive to lived spatialities. Articulating mobilityethics of relationship-dignity, the analysis of Trois femmes puissantes by Marie Ndiaye reveals how the spatiality of Africa and Europe, in migratory displacement, is construction, negotiation of meaning and liberation from original suffering.

\section{INDEX}

Mots-clés : mobilité, migration, éthique, dignité, Ndiaye

Keywords : mobility, migration, ethics, dignity, Ndiaye

\section{AUTEUR}

\section{YOUCEF IMMOUNE}

Laboratoire Études de pragmatique inférentielle, Université d'Alger 2 